\title{
Splenectomy for Splenic Abscess
}

\author{
Kathleen B. To, Laraine L. Washer, ${ }^{2}$ Oliver A. Varban, ${ }^{3}$ Jonathan W. Haft, ${ }^{4}$ \\ Amanda Fisher-Hubbard, ${ }^{5}$ and Lena M. Napolitano ${ }^{1}$
}

A 63-year-old male presented with abdominal pain and on diagnostic evaluation with computed tomography of the abdomen was noted to have a splenic infarct. The patient had a history of hypertension and repair of a right hip fracture complicated by extensive post-operative deep vein thrombosis in the right lower extremity that was treated with warfarin. Blood cultures were not obtained at the patient's initial presentation, but 2 wks later he was found to have Enterococcus faecalis bacteremia. Ultrasound once again demonstrated the splenic infarct with some fluid component. Transthoracic echocardiography confirmed mitral valve endocarditis with severe mitral regurgitation but no symptoms of heart failure. Magnetic resonance imaging of the patient's head showed a punctate, contrast-enhancing lesion in the anterior frontal lobe, raising concerning about the possibility of an asymp- tomatic brain embolus. He was treated with intravenous penicillin and gentamicin, with a plan to undergo mitral valve replacement after completion of his antibiotic regimen. However, interval abdominal ultrasound imaging revealed a splenic fluid collection consistent with a splenic abscess.

The patient was managed initially with a percutaneous drainage catheter placed into the splenic abscess by the interventional radiology service and $6 \mathrm{wks}$ of systemic antibiotic therapy. A repeat abdominal ultrasound examination demonstrated resolution of the splenic abscess, and the drainage catheter was removed. However, a week after this, while the patient was still receiving antibiotics, further imaging showed re-development of the splenic abscess (Fig. 1). The decision was therefore made to proceed with splenectomy. Vaccinations for encapsulated organisms were given

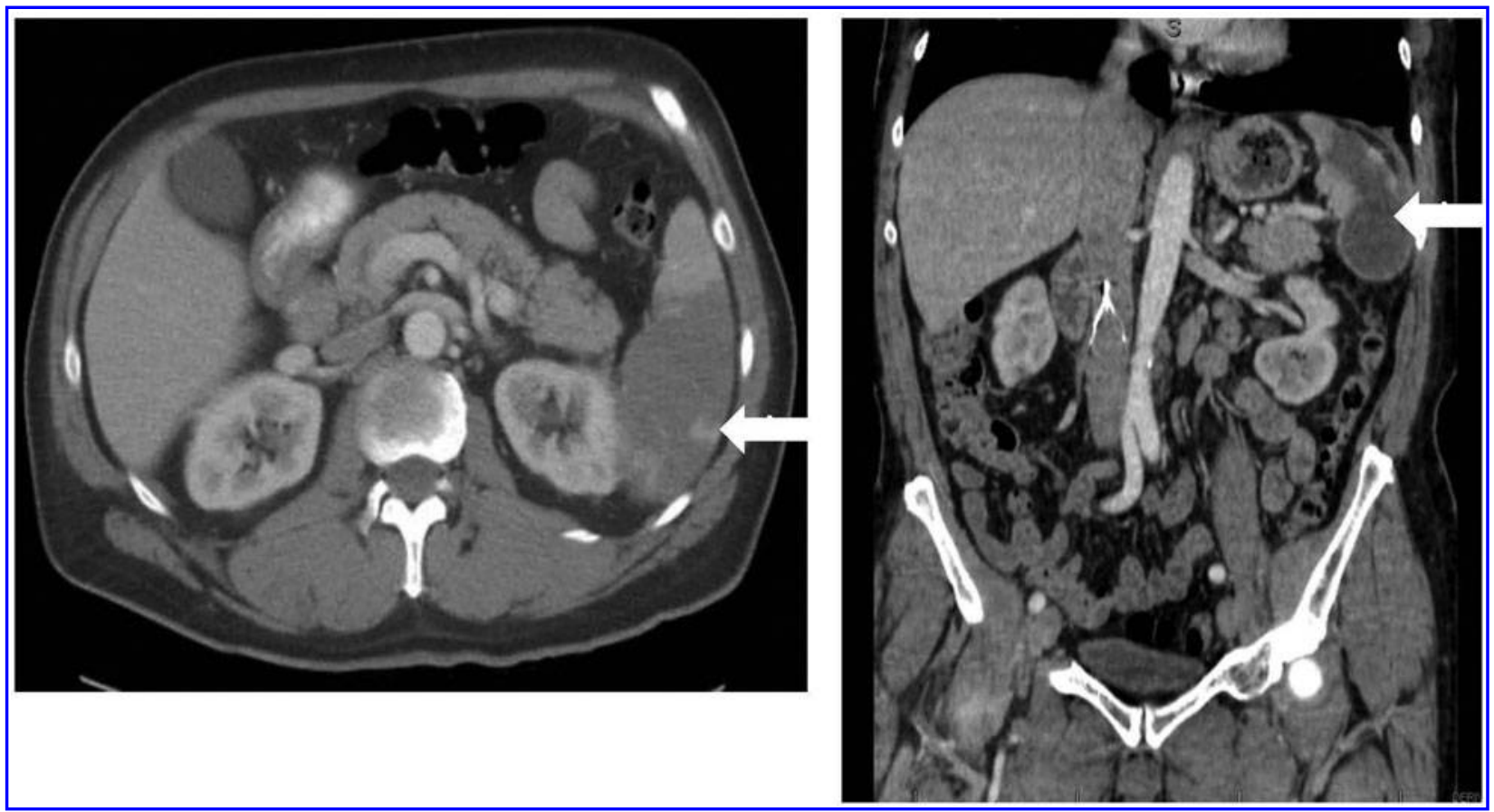

FIG. 1. Craniocaudad (left) and anteroposterior (right) computed tomographic images of splenic abscess (arrows).

Division of Acute Care Surgery, Departments of ${ }^{1,3}$ Surgery, ${ }^{2}$ Internal Medicine, ${ }^{4}$ Cardiac Surgery, and ${ }^{5}$ Pathology, University of Michigan Hospital and Health Systems, Ann Arbor, Michigan. 


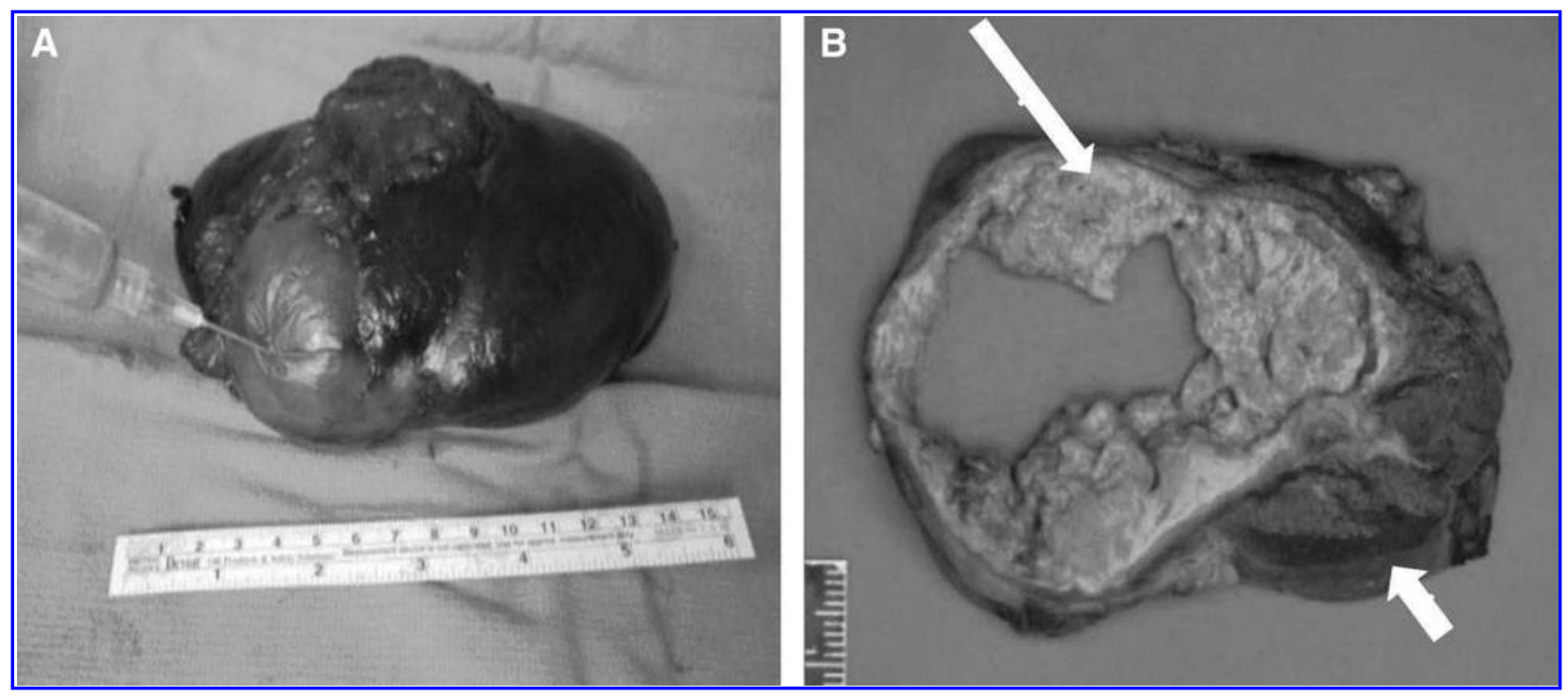

FIG. 2. (A) Surgical specimen with aspiration of abscess contents for gram stain and culture. (B) Cross-sectional gross specimen showing replacement of normal splenic parenchyma (wide arrow at bottom) by abscess cavity (small arrow at top).

pre-operatively. Because the patient had had no prior abdominal surgery, we were able to perform a hand-assisted laparoscopic splenectomy without complication. Intraoperative cultures of the patient's splenic abscess identified E. faecalis susceptible to penicillin, ampicillin, and vancomycin. Surgical pathology confirmed a multiloculated splenic abscess measuring $8.5 \times 6.5 \times 5.0 \mathrm{~cm}$ (Fig. 2). The patient went home on post-operative day three and underwent mitral valve replacement $5 \mathrm{wks}$ later. Tissue cultures of his mitral valve were negative.

Splenic abscess is an uncommon disease, usually associated with endocarditis or immunosuppression, with a reported incidence of $0.14 \%-0.7 \%$ in autopsy studies and fewer than 600 cases reported in the literature [1]. Its most common etiology is hematogenous spread of a pathogen from an infective focus elsewhere in the body. Infective endocarditis, associated with systemic embolization in $20 \%-50 \%$ of cases, is accompanied by a $10 \%-20 \%$ incidence of splenic abscess. The causative pathogens are diverse, and the condition is polymicrobial in up to $50 \%$ of cases. Treatment consists either of percutaneous drainage or splenectomy (laparoscopic or open) with appropriate concomitant antimicrobial therapy [2]. Percutaneous drainage is indicated for easily accessible abscesses with few loculations and for patients who cannot tolerate surgery with general anesthesia. Multilocular splenic abscesses, ill-defined cavities, septations, and necrotic debris typically do not respond well to percutaneous drainage. Laparoscopic splenectomy for splenic abscess can be performed safely and is associated with a decreased length of stay [3]. In cases of concurrent infective endocarditis, the decision to perform simultaneous rather than staged splenectomy and valve replacement depends on individual patient factors $[4,5]$.

\section{References}

1. Ferraioli G, Brunetti E, Gulizia R, et al. Management of splenic abscess: Report on 16 cases from a single center. Int J Infect Dis 2009;524-530.

2. Zerem E, Bergsland J. Ultrasound guided percutaneous treatment for splenic abscess: The significance of critically ill patients. World J Gastroenterol 2006;12:7341-7345.

3. Carbonell AM, Kercher KW, Matthews BD, et al. Laparoscopic splenectomy for splenic abscess. Surg Laparosc Endosc Percutan Tech 2004;289-291.

4. Simsir SA, Cheeseman SH, Lancey RA, et al. Staged laparoscopic splenectomy and a valve replacement in splenic abscess and infective endocarditis. Ann Thorac Surg 2003;75: 1635-1637.

5. Yoshikai M, Kamachi M, Kobayashi K, et al. Splenic abscess associated with active infective endocarditis. Jpn J Thorac Cardiovasc Surg 2002;50:478-480.

Address correspondence to: Dr. Kathleen B. To

Division of Acute Care Surgery Department of Surgery University of Michigan Hospital and Health Systems 1500 E. Medical Center Dr., UH 1C-340 Ann Arbor, MI 48109-5033

E-mail: kato@umich.edu 